\title{
Doxapram Treatment and Diaphragmatic Activity in Preterm Infants
}

\author{
Cornelia G. de Waal $^{\mathrm{a}}$ Gerard J. Hutten ${ }^{\mathrm{a}}$ Juliette V. Kraaijenga ${ }^{\mathrm{a}}$ \\ Frans H. de Jongh ${ }^{a}$ Anton H. van Kaam ${ }^{a, b}$ \\ aDepartment of Neonatology, Emma Children's Hospital, Academic Medical Centre Amsterdam, Amsterdam, \\ The Netherlands; ${ }^{b}$ Department of Neonatology, VU University Medical Center, Amsterdam, The Netherlands
}

\author{
Keywords \\ Doxapram · Transcutaneous electromyography · \\ Diaphragm
}

\begin{abstract}
Background: Doxapram is a treatment option for severe apnea of prematurity (AOP). However, the effect of doxapram on the diaphragm, the main respiratory muscle, is not known.

Objectives: To investigate the effect of doxapram on diaphragmatic activity measured with transcutaneous electromyography of the diaphragm (dEMG). Methods: A pilot study was conducted in a tertiary neonatal intensive care unit. Diaphragmatic activity was measured from $30 \mathrm{~min}$ before up to $3 \mathrm{~h}$ after the start of doxapram treatment. dEMG parameters were compared to baseline ( $5 \mathrm{~min}$ before doxapram treatment) and at 15, 60, 120 and 180 min after the start of doxapram infusion. Results: Eleven preterm infants were included with a mean gestational age of $25.5 \pm 1.2$ weeks and birth weight of $831 \pm 129 \mathrm{~g}$. The amplitude $\mathrm{dEMG}_{\mathrm{d}}$ peak $_{\mathrm{dEMG}}$ and tonic $\mathrm{dEMG}_{\mathrm{dEM}}$ values did not change in the $3 \mathrm{~h}$ after the start of doxapram infusion compared to baseline. Clinically, the number of apnea episodes in the $24 \mathrm{~h}$ after doxapram treatment decreased significantly. Conclusion: Doxa-
\end{abstract}

\begin{tabular}{ll}
\hline KARGER & $\begin{array}{l}\text { ( ) 2018 The Author(s) } \\
\text { Published by S. Karger AG, Basel Openger }\end{array}$ \\
E-Mail karger@karger.com & This article is licensed under the Creative Commons Attribution- \\
www.karger.com/neo & NonCommercial-NoDerivatives 4.0 International License (CC BY- \\
NC-ND) (http://www.karger.com/Services/OpenAccessLicense). \\
Usage and distribution for commercial purposes as well as any dis- \\
tribution of modified material requires written permission.
\end{tabular}

pram infusion does not alter diaphragmatic activity measured with transcutaneous dEMG in preterm infants with $A O P$, indicating that its working mechanism is primarily on respiratory drive and not on respiratory muscle activity.

(C) 2018 The Author(s)

Published by S. Karger AG, Basel

\section{Introduction}

Apnea of prematurity (AOP) is common in preterm infants and can be treated with pharmacological interventions and respiratory support $[1,2]$. First-line therapy consists of methylxanthines, i.e. caffeine, and nasal continuous positive airway pressure. When this treatment is not sufficient, doxapram could be considered [2]. Doxapram is an analeptic drug that stimulates peripheral chemoreceptors in the carotid bodies as well as the central chemoreceptors in the respiratory center in the brainstem [3]. Previous research has shown that infusion of doxapram is effective in reducing apneic events and preventing respiratory insufficiency requiring intubation and invasive mechanical ventilation [4]. Furthermore, some small physiological studies have shown an increase in 
minute ventilation and tidal volume after infusion of doxapram, but only if dosed $>1 \mathrm{mg} / \mathrm{kg} / \mathrm{h}[5,6]$. However, due to concerns about the effect of doxapram on longterm outcomes of preterm infants it is used as a rescue in infants with imminent respiratory failure due to severe AOP [7].

Recently, it has been shown that caffeine administration in preterm infants suffering from AOP leads to an increase in diaphragmatic activity [8]. However, the effect of doxapram on the diaphragm, the main respiratory muscle, is not known. Therefore, a pilot study was conducted to investigate the effect of doxapram on diaphragmatic activity measured with transcutaneous electromyography of the diaphragm (dEMG).

\section{Methods}

A prospective observational pilot study was conducted in the neonatal intensive care unit of the Academic Medical Centre Amsterdam, the Netherlands. Preterm infants born at a gestational age of less than 32 weeks with AOP unresponsive to caffeine treatment and noninvasive respiratory support were included when starting treatment with doxapram infusion. The decision to start doxapram was made by the attending physician. The study protocol was approved by the local Medical Ethics Committee, and both parents provided written informed consent.

\section{Study Procedure}

Recording of diaphragmatic activity was initiated $30 \mathrm{~min}$ before the start of doxapram treatment and was continued for $3 \mathrm{~h}$ after the infusion had started. Transcutaneous dEMG was obtained from 3 skin electrodes (disposable Kendall H59P Electrodes; Covidien, Mansfield, MA, USA), 2 placed on the left and right frontal diaphragm and 1 common electrode placed on the sternum. The electrodes were connected to a portable 16-channel physiological amplifier (Dipha-16, Demcon, Son, the Netherlands), which wirelessly sent the measured raw dEMG data to a bedside computer. The software package Polybench (Applied Biosignals, Weener, Germany) was used to process the raw signal to an averaged dEMG signal, which was used for data analyses. Further information on data processing has been published previously [9].

According to our unit protocol, doxapram infusion started with a loading dose of $2.5 \mathrm{mg} / \mathrm{kg}$ in $15 \mathrm{~min}$ and was thereafter continued with an infusion rate of $0.5 \mathrm{mg} / \mathrm{kg} / \mathrm{h}$. Depending on the effect on apnea, the dose could be increased to a maximum of 2.0 $\mathrm{mg} / \mathrm{kg} / \mathrm{h}$. Failure of doxapram treatment was defined as respiratory failure requiring intubation and invasive mechanical ventilation within $48 \mathrm{~h}$ after doxapram had been started.

\section{Data Collection and Analysis}

Demographic and clinical data were collected of all included infants. Data on respiratory support was collected up to $48 \mathrm{~h}$ after the start of doxapram. The number of apnea episodes, associated with desaturation $\left(\mathrm{SpO}_{2}<80 \%\right)$ and/or bradycardia (heart rate $<100$ beats/min), was registered in the $24 \mathrm{~h}$ before and $24 \mathrm{~h}$ after

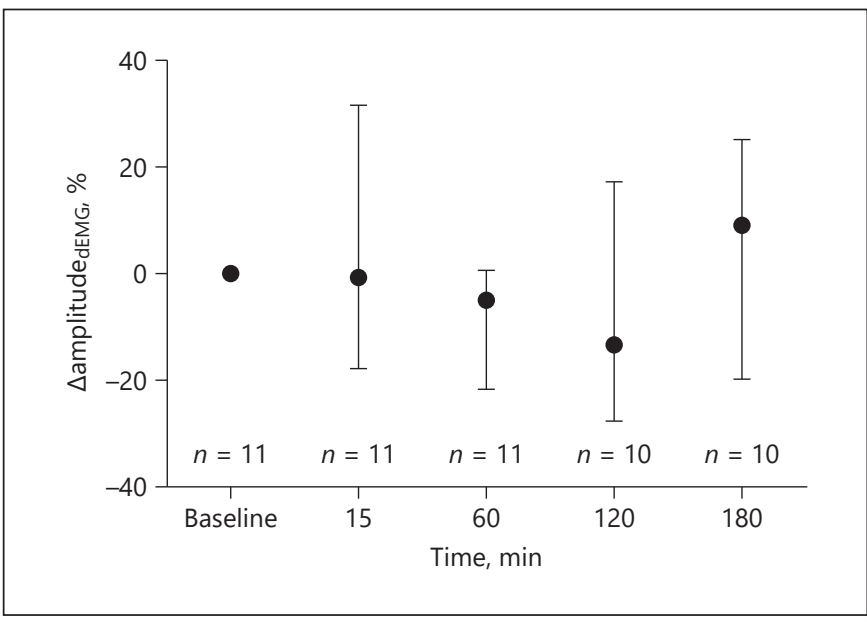

Fig. 1. Percentage change in amplitude ${ }_{\mathrm{dEMG}}$ at baseline before doxapram and in the $3 \mathrm{~h}$ thereafter. Displayed are the median and interquartile range for each time point. $\mathrm{dEMG}$, transcutaneous electromyography of the diaphragm; $n$, number of infants.

the start of doxapram infusion. Diaphragmatic activity was measured as amplitude $\mathrm{dEMG}_{\mathrm{dEM}}$, peak $\mathrm{dEMG}_{\mathrm{dEG}}$ and tonic $\mathrm{C}_{\mathrm{dEMG}}$ values of the dEMG signal over $30 \mathrm{~s}$ of stable recording at baseline ( $5 \mathrm{~min}$ before doxapram treatment) and at 15, 60, 120 and 180 min after the start of doxapram infusion. A percentage change in dEMG-derived parameters was measured at each time point compared to baseline. Furthermore, the inspiratory time, expiratory time, respiratory rate and heart rate were collected from the dEMG signal at all time points. Data on oxygenation and ventilation were not recorded as monitoring of these variables is not standard of care in our unit when infants are on noninvasive support.

\section{Statistical Analysis}

SPSS version 24 (IBM, Armonk, New York, NY, USA) was used for statistical analysis. Depending on their distribution, data were expressed as mean \pm standard deviation or median (interquartile range). Numerical data were expressed as numbers and percentages.

Repeated measurement analysis was done by using the Friedman test and post hoc Dunn test to describe dEMG and clinical parameters over time. The number of apnea episodes in the $24 \mathrm{~h}$ before and $24 \mathrm{~h}$ after the start of doxapram were compared with the Wilcoxon signed rank test. A $p$ value of $<0.05$ was defined as statistically significant.

\section{Results}

\section{Study Population}

Eleven preterm infants with a mean gestational age of $25.5 \pm 1.2$ weeks and birth weight of $831 \pm 129 \mathrm{~g}$ were measured with dEMG when doxapram infusion was started (median postnatal age: 15 days, interquartile range 
Table 1. The effect of doxapram infusion on parameters measured with transcutaneous dEMG

\begin{tabular}{|c|c|c|c|c|c|c|}
\hline dEMG parameters & $\begin{array}{l}\text { Baseline } \\
(n=11)\end{array}$ & $\begin{array}{l}15 \min \\
(n=11)\end{array}$ & $\begin{array}{l}60 \min \\
(n=11)\end{array}$ & $\begin{array}{l}120 \text { min } \\
(n=10)\end{array}$ & $\begin{array}{l}180 \min \\
(n=10)\end{array}$ & $p$ value \\
\hline$\Delta$ Peak $_{\mathrm{dEMG}}, \%$ & $0(0$ to 0$)$ & 1.1 (-10.8 to 20.7$)$ & $-5.4(-28.05$ to 2.8$)$ & $-9.1(-27.8$ to 3.9$)$ & $-6.6(-18.0$ to 27.4$)$ & 0.811 \\
\hline$\Delta$ Tonic $_{\mathrm{dEMG}}, \%$ & $0(0$ to 0$)$ & $2.7(-12.0$ to 17.6$)$ & $-6.4(-19.4$ to 28.7$)$ & $-5.8(-24.7$ to 4.2$)$ & $14.7(-23.2$ to 32.8$)$ & 0.293 \\
\hline Inspiratory time, $s$ & $0.5(0.4$ to 0.5$)$ & $0.5(0.4$ to 0.5$)$ & $0.5(0.4$ to 0.5$)$ & $0.5(0.4$ to 0.5$)$ & $0.5(0.4$ to 0.5$)$ & 0.988 \\
\hline Expiratory time, s & $0.6(0.4$ to 0.6$)$ & $0.6(0.5$ to 0.6$)$ & $0.5(0.5$ to 0.6$)$ & $0.6(0.5$ to 0.7$)$ & $0.5(0.5$ to 0.6$)$ & 0.993 \\
\hline Respiratory rate, breaths/min & $56(53$ to 77$)$ & $58(53$ to 64$)$ & $61(57$ to 67$)$ & $58(52$ to 64$)$ & $61(54$ to 67$)$ & 0.949 \\
\hline
\end{tabular}

Peak $_{\mathrm{dEMG}}$ and tonic $\mathrm{dEMG}_{\mathrm{dEM}}$ values are expressed as median percentage change compared to baseline (IQR). $p$ values based on the Friedman test for repeated measurements. dEMG, electromyography of the diaphragm.

11-22). All infants were treated with caffeine base (5 mg/ $\mathrm{kg} /$ day) and nasal intermittent positive pressure ventilation at the time doxapram was started. $82 \%$ of the infants had been mechanically ventilated before doxapram treatment.

\section{The Effect of Doxapram}

The amplitude $\mathrm{dEMG}_{\mathrm{d}}$, peak $\mathrm{dEMG}_{\mathrm{dEM}}$ and tonic $\mathrm{dEMG}_{\mathrm{dEM}}$ values did not change in the $3 \mathrm{~h}$ after the start of doxapram infusion compared to baseline (Fig. 1; Table 1). Furthermore, no change was seen in inspiratory time, expiratory time, respiratory rate and heart rate in this time window (Table 1).

Four (36\%) infants failed doxapram treatment and needed invasive mechanical ventilation within $48 \mathrm{~h}$. Compared to infants successfully treated with doxapram, no difference was found in terms of diaphragmatic response.

Clinically, the treatment with doxapram led to a significant decrease in the number of apnea episodes from 32 (interquartile range 24-51) in the $24 \mathrm{~h}$ before to 23 (interquartile range 18-28) in the $24 \mathrm{~h}$ after the start ( $p=$ 0.012 ). This reduction in apnea was most prominent in infants not requiring intubation and invasive mechanical ventilation compared to infants who failed doxapram treatment and needed invasive mechanical ventilation within $48 \mathrm{~h}$ after starting doxapram treatment.

\section{Discussion}

In this pilot study on the effect of doxapram on diaphragmatic activity in preterm infants, no significant change in dEMG-derived parameters was found in the first $3 \mathrm{~h}$ after the start of doxapram treatment.
Doxapram is an increasingly used drug to treat infants with severe AOP resistant to caffeine therapy and maximal noninvasive respiratory support [10]. Previous studies have shown that doxapram reduces apnea frequency and the need for invasive mechanical ventilation [4]. However, a recent systematic review, based on a small number of studies with mainly low quality of evidence, indicated that no firm conclusions can be drawn on the efficacy and safety of doxapram [7]. Furthermore, its working mechanism is still poorly understood.

Our study confirms the positive effect of doxapram on apnea reduction but adds important new knowledge that this beneficial effect is probably not mediated through an increase in diaphragmatic activity measured in stable transcutaneous $\mathrm{dEMG}$ recordings. This suggests that the primary effect of doxapram is to regulate respiratory drive and not respiratory muscle activity. This is in contrast to caffeine, which results in a direct and persistent increase in diaphragmatic activity in preterm infants [8]. It is unlikely that this different effect of doxapram and caffeine on diaphragmatic activity is dose related, as we administered a doxapram loading dose of $2.5 \mathrm{mg} / \mathrm{kg}$ in 15 min which is the maximal tested dose for treatment of AOP in preterm infants [11]. Therefore, the working mechanism of doxapram is actually different from that of caffeine which makes a synergistic effect of these drugs possible. As doxapram is very rapidly metabolized and its clinical effect is often seen within minutes, extending the 3-h measurement would probably not have changed our findings. It is important to acknowledge that we only included 11 infants in this physiological study. However, the absence of any signal indicating a doxapram-mediated effect on diaphragmatic activity makes it unlikely that we would have found a clinically relevant treatment effect on this outcome in a larger sample size. Furthermore, 
the sample size used in this study is common in this type of research.

In conclusion, doxapram infusion does not alter diaphragmatic activity measured with transcutaneous dEMG in preterm infants with AOP, indicating that its working mechanism is primarily on respiratory drive and not on respiratory muscle activity. This probably explains the additive effect of doxapram in caffeine-treated preterm infants with persistent AOP.

\section{Statement of Ethics}

The study protocol was approved by the local Medical Ethics Committee, and both parents provided written informed consent.

\section{Disclosure Statement}

Each author listed on the manuscript has no potential, real or perceived conflicts of interest in the study. Miss C.G. de Waal wrote the first draft of the manuscript.

No financial assistance (honorarium, sponsorship, grant or other form of payment) was received in support of the study.

\section{References}

1 Eichenwald EC. Apnea of Prematurity. Pediatrics. 2016 Jan;137(1):e20153757.

2 Hascoet JM, Hamon I, Boutroy MJ. Risks and benefits of therapies for apnoea in premature infants. Drug Saf. 2000 Nov;23(5):363-79.

3 Yost CS. A new look at the respiratory stimulant doxapram. CNS Drug Rev. 2006;12(3-4): 236-49.

4 Flint R, Halbmeijer N, Meesters N, van Rosmalen J, Reiss I, van Dijk M, et al. Retrospective study shows that doxapram therapy avoided the need for endotracheal intubation in most premature neonates. Acta Paediatr. 2017 May;106(5):733-9.
5 Barrington KJ, Finer NN, Peters KL, Barton J. Physiologic effects of doxapram in idiopathic apnea of prematurity. J Pediatr. 1986 Jan; 108(1):124-9.

6 Bairam A, Faulon M, Monin P, Vert P. Doxapram for the initial treatment of idiopathic apnea of prematurity. Biol Neonate. 1992; 61(4):209-13.

7 Vliegenthart RJ, Ten Hove CH, Onland W, van Kaam AH. Doxapram Treatment for Apnea of Prematurity: A Systematic Review. Neonatology. 2017;111(2):162-71.

8 Kraaijenga JV, Hutten GJ, de Jongh FH, van Kaam AH. The Effect of Caffeine on Diaphragmatic Activity and Tidal Volume in Preterm Infants. J Pediatr. 2015 Jul;167(1): $70-5$.
9 O’Brien M, van Eykern L, Prechtl H. Monitoring respiratory activity in infants - A non-intrusive diaphragm EMG technique. Non-invasive physiological measurements. London: Academic Press; 1983. p. 131-77.

10 Prins SA, Pans SJ, van Weissenbruch MM, Walther FJ, Simons SH. Doxapram use for apnoea of prematurity in neonatal intensive care. Int J Pediatr. 2013;2013:251047.

11 Barrington KJ, Finer NN, Torok-Both G, Jamali F, Coutts RT. Dose-response relationship of doxapram in the therapy for refractory idiopathic apnea of prematurity. Pediatrics. 1987 Jul;80(1):22-7. 